\title{
OJ 287: A BLAZAR WITH EVERYTHING
}

\author{
L.O. TAKALO \\ Tuorla Observatory, Tuorla, \\ 21500 Piikkiö, Finland
}

\begin{abstract}
.
We have collected all the observations available to us of blazar OJ 287. Here we will present preliminary results from our investigation of these data. The photometric light curves show large outbursts occuring (quasi)simultaneously in all frequences. The largest outbursts occured during 1972 and 1983. The B-band light curve can be extended to the year 1894. This being the longest available observational data set of all blazars. Polarization light curves in optical show random variations in all timescales. In the radio bands the polarization observations show well defined structure in the light curves. Light curves and simple correlation analysis based on the light curves on different frequences will be presented.
\end{abstract}

OJ 287 is one of the best observed blazars, and also one of the most exiting ones. It shows all the characteristic blazar behaviours, but it has some features that are observed only in it. One of these unigue features are the observed periodic variations, in time scales from tens of minutes to years.

We have collected all the available data on OJ 287 with the intention of doing a "complete" study of its behaviour and comparison at different wavelenghts.

The historical light curve clearly shows outbursts at 1913, 1937, 1947, 1959, 1972 and 1983. Based on these data several groups have calculated that the outbursts occur with a 11.6 year period. OJ 287 is the only blazar, in which this kind of periodicity is so well observed. Based on this periodicity one can predict that the next outburst should occur during 1994!

Comparing the light curves of the last two outbursts (1972 and 1983) indicate very similar behaviour, with the double maxima and slow decline with small (periodic) variations. The time separation between the two maxima is about one and a half years.

The polarization is seen to show random variations in time scales from days to months. During the 1983 outburst the polarization level was almost zero. But right after the outburst it was over $30 \%$. The "average" polarization seems to around $10 \%$. Position angle behaviour is really remarkable. Around the average position angle of 90 degrees the variations are fast and large. No preferred position angle can be seen.

The variability has smaller amplitude in the radio bands than in the optical, and the 1983 outburts is not so noticeable. The polarization level is smaller than the optical one (average being $5 \%$ ). The average position angle is around 100 degrees, similar to the optical one.

We have presented preliminary results of a "complete" study of blazars OJ 287 in all frequences. OJ 287 show (periodic) variability in all frequences in time scales from minutes to years. Being the only blazar with this kind of behavior.

T. J.-L. Courvoisier and A. Blecha: Multi-Wavelength Continuum Emission of AGN, 409.

(C) 1994 IAU. Printed in the Netherlands. 